\title{
Exploración Inicial de la Estructura Genética del Cerdo Doméstico (Sus scrofa domestica) en Sampués, Sucre, Colombia, utilizando Microsatélites
}

\author{
Initial Exploration of the Genetic Structure of Domestic Pig (Sus scrofa \\ domestica) in Sampués, Sucre, Colombia, using Microsatellites
}

Enrique Pardo P. ${ }^{1}$, Alfonso Calderón R. ${ }^{2}$, Guillermo Arrazola P. ${ }^{3}$

\section{Resumen}

El objetivo de la presente investigación fue evaluar la variabilidad genética de una población de cerdo doméstico (Sus scrofa domestica) en Sampués, Sucre, Colombia, para determinar su situación genética. Se estudiaron 50 muestras de la población. Se utilizaron 20 microsatélites, cinco pertenecientes a la lista de los recomendados por la FAO/ISAG para estudios de biodiversidad porcina y los restantes representan la mayor parte del genoma porcino. Se pudo precisar que los microsatélites utilizados resultaron polimórficos, detectándose entre 3 (SW2019) y 14 (SW957) alelos, con un número medio de alelos de 6 y un total de 120 . La heterocigosidad media esperada fue de 0.5465 y la heterocigosidad media observada fue de 0.5203 . Los valores del contenido de información polimórfica (PIC) oscilaron entre 0.2823 y 0.7252 para los loci $S W 1041$ y $S W 957$, respectivamente. Los resultados muestran a la población de cerdos estudiada como un grupo con alto grado de diversidad genética.

Palabras clave: marcadores microsatélites, Sus scrofa domestica, diversidad, equilibrio Hardy-Weinberg, Sampués

\section{Abstract}

The aim of this research was to evaluate the genetic variability of a population of domestic pigs (Sus scrofa domestica) in Sampués, Sucre, Colombia to determine their genetic status. Fifty samples were studied. Twenty microsatellites were used where five of them were from the list of those recommended by FAO/ISAG for studies of swine biodiversity and the remaining represent most of the pig genome. The microsatellites

\footnotetext{
${ }^{1}$ Departamento de Biología, Facultad de Ciencias Básicas, Universidad de Córdoba, Colombia

${ }^{2}$ Departamento de Ciencias Pecuarias, Facultad de Medicina Veterinaria y Zootecnia, Universidad de Córdoba, Colombia

${ }^{3}$ Programa de Ingeniería de Alimentos, Facultad de Ingenierías, Universidad de Córdoba, Colombia

${ }^{4}$ E-mail: epardop@correo.unicordoba.edu.co
}

Recibido: 8 de julio de 2016

Aceptado para publicación: 14 de diciembre de 2016 
used were polymorphic, detecting between 3 (SW2019) to 14 (SW957) alleles with an average 6 and a total of 120 . The mean expected heterozygosity was 0.5465 and the mean observed heterozygosity was 0.5203 . The polymorphism information content (PIC) ranged between 0.2823 and 0.7252 for $S W 1041$ and $S W 957$ loci respectively. The results showed that the studied population as a group with a high degree of genetic diversity.

Key words: microsatellite markers, Sus scrofa domestica, diversity, Hardy-Weinberg equilibrium, Sampues

\section{INTRODUCCIÓN}

El cerdo pertenece al orden Artiodactyla, familia Suidae y género Suis. Apareció durante el Mioceno, hace 25 a 600 millones de años, originándose a partir del jabalí (Sus scrofa), y fue domesticado hace unos 10000 años (Giuffra et al., 2000). Tanto la evidencia arqueológica como la evidencia molecular muestran que los cerdos domésticos se originaron independientemente en varias partes del mundo, a partir de múltiples subespecies de jabalíes europeos y asiáticos (Kijas y Anderson, 2001; Larson et al., 2005). De otro lado, los cerdos silvestres y domésticos pertenecen a la misma especie (Sus scrofa), el primero de los cuales tiene 36 cromosomas y el doméstico tiene 38 (Bosma, 1976).

El cerdo, Sus scrofa, es una especie foránea al continente americano. Inicialmente llegaron a las Antillas y desde allí emigraron al resto del continente. Colón trajo cerdos en su segundo viaje (Burgos et al., 2013). Años después, y por exigencia de Carlos V, la excursión de Rodrigo Bastidas que salió de la Española y fundó Santa Marta en 1525, trajo 300 cerdos (Peña y Mora, 1977). Es posible que dichos cerdos hayan sido los primeros que llegaron a Colombia.

El departamento de Sucre es una de las regiones de Colombia con menor población de cerdo doméstico (Sus scrofa domestica). Presentó una población estimada de 124455 ejemplares en 2016, de los cuales, el 96.4\% eran de crianza de traspatio (Ministerio de Agricultura, 2016), siendo mayormente de tipo criollo y cruces con diversas razas.

La reacción en cadena de la polimerasa (PCR) es una técnica enzimática in vitro, utilizada para amplificar exponencialmente una región determinada de $\mathrm{ADN}$, cuya secuencia se conoce, a partir de una mezcla compleja de ácidos nucleicos. Es la técnica más empleada para el estudio de marcadores polimórficos como son los microsatélites o SSRs (Simple Sequence Repeats), formados por secuencias de ADN constituidas por repeticiones de motivos nucleotídicos de $1 \mathrm{a}$ 6 pares de bases repetidas en tándem (Hancock, 1999), Son marcadores neutrales de gran valor informativo, que constituyen una poderosa herramienta para estudios genéticos, los cuales han sido utilizados para estudios de caracterización y diversidad genética, relaciones genéticas entre poblaciones, influencia de una raza sobre otra (admixture), pruebas de paternidad, consanguinidad y cuellos de botella genéticos, entre otros (Quiroz et al., 2007).

Los objetivos del presente estudio fueron identificar el estado de la diversidad genética de la población de cerdos domésticos en Sampués, Sucre, mediante la utilización de 20 microsatélites, calcular heterocigosidades por locus y contrastar dicha información con la obtenida en otras poblaciones con los mismos marcadores genéticos. 


\section{Materiales Y MéTOdos}

Se recolectaron muestras de pelo de 50 cerdos domésticos, procedentes de explotaciones familiares de Sampués, departamento de Sucre, Colombia ( $9^{\circ} 11^{\prime} 0.8^{\prime \prime}$ latitud Norte y $75^{\circ} 22^{\prime} 51^{\prime \prime}$ longitud Oeste). Se desconoce el registro genealógico de los cerdos.

El ADN se extrajo de las muestras mediante una modificación al protocolo descrito por Sambrook y Russell (2001), a partir del bulbo piloso, mediante digestión de proteínas con la enzima Proteinasa K y una purificación con fenol-cloroformo.

Se utilizaron 20 microsatélites, cinco de los cuales pertenecen a la lista recomendada por FAO/ISAG (FAO, 2011) para estudios de biodiversidad porcina y los restantes representan la mayor parte del genoma porcino (Cuadro 1). La amplificación de los mismos se efectuó utilizando un termociclador Mycycler Bio-Rad ${ }^{\circledR}$ en un volumen final de $25 \mathrm{ml}$ que incluyó 10 il de dNTPs $100 \mathrm{iM}, 2.5$ il de amortiguador $10 \mathrm{X}, 1.0$ il de $\mathrm{MgCl}_{2} 25$ $\mathrm{mM}, 3.0$ il de cebadores específicos de cada locus de 10 pmol, 0.3 il de enzima Taq ADN polimerasa (Invitrogen ${ }^{\circledR}$ ) a una concentración de $1 \mathrm{U} / \mathrm{il}, 4.0$ il de $\mathrm{ADN}$ genómico a una concentración de $50 \mathrm{ng} / \mathrm{il} \mathrm{y} 4.2$ ìl de agua bidestilada esterilizada.

La reacción de PCR consistió de una fase de desnaturalización a $94{ }^{\circ} \mathrm{C}$ por $30 \mathrm{~s}$, seguida de 30 ciclos de desnaturalización a $94^{\circ} \mathrm{C}$ por $30 \mathrm{~s}$, hibridización a $56^{\circ} \mathrm{C}$ por $30 \mathrm{~s}$ y elongación a $72^{\circ} \mathrm{C}$ por $30 \mathrm{~s}$. Los productos de PCR se separaron mediante electroforesis vertical en gel de poliacrilamida en una cámara Mini-Protean II Biorad ${ }^{\circledR}$. Las bandas se visualizaron por tinción con nitrato de plata (Shanlian et al., 2012).

La determinación del tamaño alélico se efectuó mediante la escalera alélica, y la asignación alélica se realizó mediante el ajuste a una curva de regresión lineal desarrollada a partir de las distancias de migración de los fragmentos de tamaño conocido.
Las frecuencias alélicas, heterocigosidades, valor de $\mathrm{F}_{\text {IS }}$ (Weir, 2012), existencia de equilibrio Hardy-Weinberg (HW), riqueza alélica y coeficiente de consanguinidad se evaluaron mediante el programa GENEPOP v. 4.0.6 (Rousset, 2008). Adicionalmente, se calculó el Contenido de Información Polimórfica (PIC) para medir la diversidad alélica de los microsatélites (Botstein et al., 1980) mediante el programa CERVUS v. 3.0.3 (Kalinowski et al., 2007).

\section{Resultados}

Los loci de los 20 microsatélites fueron polimórficos detectándose un total de 120 alelos. El número de alelos por locus varió entre 3 (SW2019) y 14 (SW957) (Cuadro $1)$, con un valor medio para todos los loci de 6 alelos.

Los valores de PIC (Cuadro 2) fluctuaron entre 0.2823 (SW2019) y 0.7252 (SW957), correspondiendo estos valores con los marcadores que presentaron el menor y el mayor número de alelos. El PIC promedio encontrado fue de 0.5478 .

La heterocigosidad esperada (Cuadro 2), varió entre 0.1164 para $S W 1067$ y 0.8583 para el marcador $S W 957$, con una media de 0.5465 . La heterocigosidad observada (Cuadro 2) varió entre 0.0241 para $S W 1067$ y 0.8638 para el microsatélite $S W 2519$, con una media de 0.5203 . La heterocigosidad esperada fue un poco mayor que la heterocigosidad observada.

El test de equilibrio Hardy-Weinberg indicó que 15 microsatélites se encontraron en equilibrio de Hardy-Weinberg y cinco loci mostraron una desviación significativa con respecto al equilibrio H-W (SW2019, S0215, $S W 72, S 0385, S W 1067)$.

El estadístico $\mathrm{F}_{\text {IS }}$ (Cuadro 2) fluctuó entre -0.404 para $S W 911$ y 0.387 para el marcador SW1067. Doce de los 20 marca- 
Cuadro 1. Microsatélites, número de alelos detectados (NA), repetición y rango alélico (pb) del cerdo doméstico en Sampués (Sucre, Colombia)

\begin{tabular}{|c|c|c|c|}
\hline Marcador & NA & PCR Cebadores & Rango alélico (pb) \\
\hline SW489 & 6 & $\begin{array}{l}\text { CAAGTGTGAAATTTGTGCGG } \\
\text { CGAAGTGCTAACTATAAGCAGCA }\end{array}$ & $148-181$ \\
\hline$S W 2519$ & 10 & $\begin{array}{l}\text { CGTCTTCCCAGTAGGCTTTG } \\
\text { GGATACTAAGTGTCTCCCCCC }\end{array}$ & $187-232$ \\
\hline$S W 780$ & 5 & $\begin{array}{l}\text { TCTACCAGCTAAATTGCTCACTG } \\
\text { TAGGACCTGGAATATACTCCCTG }\end{array}$ & $115-170$ \\
\hline$S W 2083$ & 4 & $\begin{array}{l}\text { AAATTTTGTGAGTTTTGTGTGGG } \\
\text { ACACCTGAGAGTGTGTCTTGTAGC }\end{array}$ & $143-167$ \\
\hline SW2019 & 3 & $\begin{array}{l}\text { ATGATGCGAACCTGGAACTC } \\
\text { TATGTGTAACTTGGTCCCATGC }\end{array}$ & $127-147$ \\
\hline$S W 2410$ & 5 & $\begin{array}{l}\text { ATTTGCCCCCAAGGTATTTC } \\
\text { CAGGGTGTGGAGGGTAGAAG }\end{array}$ & $103-137$ \\
\hline S0215 & 6 & $\begin{array}{l}\text { TAGGCTCAGACCCTGCTGCAT } \\
\text { TGGGAGGCTGAAGGATTGGGT }\end{array}$ & $125-194$ \\
\hline$S W 72$ & 6 & $\begin{array}{l}\text { ATCAGAACAGTGCGCCGT } \\
\text { TTTGAAAATGGGGTGTTTCC }\end{array}$ & $97-119$ \\
\hline SW911 & 5 & $\begin{array}{l}\text { CTCAGTTCTTTGGGACTGAACC } \\
\text { CATCTGTGGAAAAAAAAAGCC }\end{array}$ & $147-177$ \\
\hline$I F N G$ & 6 & $\begin{array}{l}\text { TGTTCAGTGGGTTAAGGATCG } \\
\text { TTCCCTACACCCTGCCTTC }\end{array}$ & $221-245$ \\
\hline SW1041 & 4 & $\begin{array}{l}\text { ATCAGAAAATGGTCAACAGTTCA } \\
\text { GGAGAATTCCCAAAGTTAATAGG }\end{array}$ & $93-101$ \\
\hline SWR345 & 5 & $\begin{array}{l}\text { AACAGCTCCGATTCAACCC } \\
\text { TACTCAGCCTTAAAAGGAAGGG }\end{array}$ & $134-160$ \\
\hline$T N F B$ & 9 & $\begin{array}{l}\text { TCCTTCTTCTCTCCCCAACAGTTT } \\
\text { CTCAGCGAGTCCTCCTCATACTATC }\end{array}$ & $142-203$ \\
\hline S0385 & 6 & $\begin{array}{l}\text { CTATTAGGAGGGTTG } \\
\text { AGTTCAGAAGCTGTTGCT }\end{array}$ & $145-192$ \\
\hline SW787 & 5 & $\begin{array}{l}\text { CTGGAGCAGGAGAAAGTAAGTTC } \\
\text { GGACAGTTACAGACAGAAGAAGG }\end{array}$ & $144-164$ \\
\hline$S 0090$ & 5 & $\begin{array}{l}\text { CCAAGACTGCCTTGTAGGTGAATA } \\
\text { GCTATCAAGTATTGTACCATTAGG }\end{array}$ & $227-251$ \\
\hline SW1083 & 6 & $\begin{array}{l}\text { CCTTGCTGGCCTCCTAAC } \\
\text { CATACTCCAAAATTTCTATGTTGA }\end{array}$ & $108-152$ \\
\hline SW957 & 14 & $\begin{array}{l}\text { AGGAAGTGAGCTCAGAAAGTGC } \\
\text { ATGGACAAGCTTGGTTTTCC }\end{array}$ & $112-157$ \\
\hline SW2427 & 6 & $\begin{array}{l}\text { GCATGTTATTGAGTTGATGTGTAGG } \\
\text { TCGGAATTCCAGAAAATTGG }\end{array}$ & $116-146$ \\
\hline SW1067 & 4 & $\begin{array}{l}\text { TGCTGGCCAGTGACTCTG } \\
\text { CCGGGGGATTAAACAAAAAG }\end{array}$ & $137-175$ \\
\hline
\end{tabular}


Cuadro 2. Microsatélites tipificados, Heterocigosidad esperada (He), Heterocigosidad observada (Ho), Contenido de información polimórfica (PIC), Valores de probabilidad por desviación del equilibrio de Hardy-Weinberg e Índice de fijación $\mathrm{F}_{\mathrm{IS}}$ del cerdo doméstico de Sampués (Sucre)

\begin{tabular}{lccccc}
\hline Marcador & $\mathrm{He}$ & $\mathrm{Ho}$ & $\mathrm{PIC}$ & $\mathrm{HW}(\mathrm{p}$ value $)$ & $\mathrm{F}_{\mathrm{IS}}$ \\
\hline SW489 & 0.5321 & 0.6449 & 0.5523 & $0.3823 \mathrm{~ns}$ & 0.253 \\
SW2519 & 0.7453 & 0.8638 & 0.6826 & $0.3182 \mathrm{~ns}$ & -0.192 \\
SW780 & 0.3223 & 0.2497 & 0.5221 & $0.2982 \mathrm{~ns}$ & -0.224 \\
SW2083 & 0.5624 & 0.4535 & 0.3357 & $0.0643 \mathrm{~ns}$ & -0.132 \\
SW2019 & 0.3674 & 0.2513 & 0.2823 & $0.0032 *$ & -0.283 \\
SW2410 & 0.6793 & 0.3714 & 0.5815 & $0.1137 \mathrm{~ns}$ & 0.177 \\
S0215 & 0.6017 & 0.5685 & 0.2947 & $0.0026 *$ & -0.072 \\
SW72 & 0.2571 & 0.7636 & 0.6698 & $0.0012 *$ & -0.361 \\
SW911 & 0.6867 & 0.7314 & 0.5628 & $0.6154 \mathrm{~ns}$ & -0.404 \\
IFNG & 0.5572 & 0.5488 & 0.5021 & $0.3442 \mathrm{~ns}$ & 0.175 \\
SW1041 & 0.7159 & 0.2365 & 0.5380 & $0.1834 \mathrm{~ns}$ & -0.368 \\
SWR345 & 0.5830 & 0.5927 & 0.5133 & $0.3976 \mathrm{~ns}$ & -0.335 \\
TNFB & 0.2539 & 0.2425 & 0.3451 & $0.1211 \mathrm{~ns}$ & 0.134 \\
S0385 & 0.5938 & 0.6916 & 0.6142 & $0.0007 *$ & 0.289 \\
SW787 & 0.5356 & 0.7321 & 0.7055 & $0.2164 \mathrm{~ns}$ & -0.212 \\
S0090 & 0.6504 & 0.6541 & 0.5186 & $0.3672 \mathrm{~ns}$ & 0.126 \\
SW1083 & 0.5544 & 0.4072 & 0.6867 & $0.1277 \mathrm{~ns}$ & -0.128 \\
SW957 & 0.8583 & 0.6971 & 0.7252 & $0.2219 \mathrm{~ns}$ & -0.307 \\
SW2427 & 0.7574 & 0.6816 & 0.6583 & $0.2293 \mathrm{~ns}$ & 0.224 \\
SW1067 & 0.1164 & 0.0241 & 0.6659 & $0.0016 *$ & 0.387 \\
\hline & 0.5465 & 0.5203 & 0.5478 & & -0.0627 \\
\hline
\end{tabular}

ns: No significativo

* Marcadores que no están en equilibrio Hardy-Weinberg $(p<0.05)$

dores presentan signo positivo y ocho presentan signo negativo. El $\mathrm{F}_{\text {IS }}$ promedio fue de 0.0627 .

\section{Discusión}

Los resultados revelan un elevado nivel de polimorfismo, demostrado en la cantidad y el promedio de alelos por locus obtenido.
Otros estudios de diversidad genética en cerdo reportan valores mayores como Pham et al. (2014) entre 9 y 20 alelos y un número medio de alelos de 15.1, así como valores menores como Chang et al. (2009) entre $2 \mathrm{y}$ 6 alelos y un número medio de 3.7 alelos, Zaman et al. (2015) entre 2 y 11 alelos y un número medio de 6.5 alelos, y Cortés et al. (2016) entre 3 y 8 alelos y un número medio de 5.2 alelos. 
Según Botstein et al. (1980), 16 de los 20 marcadores utilizados pueden ser considerados muy informativos ( $\mathrm{PIC}>0.5$ ) para determinar la variabilidad genética en la población del cerdo doméstico en Sampués. Asimismo, cuatro marcadores son medianamente informativos (PIC entre 0.25 y 0.5 ). El valor medio de PIC en el presente estudio, resultó menor en comparación con datos previamente publicados en estudios realizado con cerdos de razas vietnamitas, indios y polacos (Pham et al., 2014; Zaman et al., 2015; Szmato ${ }^{3}$ a et al., 2016, respectivamente), similar a los reportados por estudios en cerdos nativos de China (Zhang et al., 2003) y mayor al reportado por Chang et al. (2009) y Oh et al. (2014).

La proporción de individuos heterocigotos detectados en el presente estudio fue mayor al $50 \%$, lográndose valores de $54.65 \%$ para la heterocigosidad media esperada y $52.03 \%$ para la heterocigosidad media observada (Cuadro 2), revelando un alto grado de variabilidad, evento que se considera ocurre, cuando los valores superan el 0.5 . Este valor es menor a otros reportes (Pham et al., 2014; Zaman et al., 2015; Cortés et al., 2016; Szmatoª et al., 2016), similar a lo hallado por Zhang et al. (2003) y mayor al reportado por Chang et al. (2009) con un He de 0.375 y Oh et al. (2014) con un He de 0.439 .

De los microsatélites examinados, 15 estaban en equilibrio de Hardy-Weinberg, lo cual muestra genéticamente estable a la población (Cuadro 2), y esto podría revelar que los cruzamientos en la población se originaron de forma azarosa, o si hay animales nuevos que se han añadido últimamente, estos descienden de poblaciones con igual acervo genético a los individuos de la población analizada (Wang, 2012). Cinco marcadores (SW2019, S0215, SW72, S0385, SW1067) mostraron desviación significativa respecto al equilibrio $\mathrm{H}-\mathrm{W}$, revelando un exceso de homocigotos, lo cual podría ser el resultado de eventos de endogamia al interior de la misma (Allendorf et al., 2012). No obstante, la endogamia afecta por igual todo el genoma por lo que se esperaría que si este fenómeno fuera el más trascendente, todos los marcadores utilizados deberían mostrar un exceso de homocigotos, cosa que no ocurre. Otra explicación para esta desviación podría ser la existencia del efecto Wahlund, lo cual mostraría la existencia de diferencias entre poblaciones contiguas de cerdo doméstico para los marcadores (SW2019, S0215, SW72, S0385, SW1067), pero no para los otros marcadores. La permanencia de las diferencias en estos microsatélites podría darse por el limitado flujo génico entre poblaciones cercanas. Asimismo, pudiera acontecer que los marcadores (SW2019, S0215, SW72, S0385, SW1067) se encuentran ligados a genes influenciados por selección natural. También puede darse la presencia de alelos nulos en dichos loci (Chapuis y Estoup, 2007), suceso descartable en este estudio por no haberse encontrado. Por último, pudo haber ocurrido un efecto fundador (arribaron pocos sementales que se reprodujeron mucho).

Los cálculos del estadístico $\mathrm{F}_{\text {IS }}$ para los 20 microsatélites, muestran ocho marcadores con signo positivo, revelando exceso de homocigotos, y 12 con signo negativo. El $\mathrm{F}_{\text {IS }}$ promedio de -0.0627 revela un bajo valor de exogamia, pero con un ligero exceso de heterocigotos. Los valores riqueza alélica y número medio de alelos por locus indican que dicha población muestra cierto grado de variabilidad.

Al no existir en Sampués, Sucre, reportes sobre la diversidad genética de Sus scrofa domestica, no se puede excluir la probabilidad de endogamia en esta población del Caribe colombiano.

\section{Conclusiones}

Los resultados permiten inferir que los microsatélites utilizados en la población de cerdo doméstico (Sus scrofa domestica) en Sampués, Sucre, Colombia, muestran un alto 
grado de polimorfismo. Igualmente, el gran número de marcadores con un PIC alto, posibilitan perfeccionar e implementar esta técnica para otros estudios dentro de la raza como la exploración genealógica y la asignación de individuos a poblaciones. Asimismo, los niveles de heterocigosidad esperada y observada revelan que muestra un alto grado de variabilidad genética.

\section{Literatura Citada}

1. Allendorf W, Luikart G, Aitken SN. 2012. Conservation and the genetics of populations. $2^{\text {nd }}$ ed. Massachusetts: Wiley-Blackwell. $624 \mathrm{p}$.

2. Bosma A. 1976. Chromosomal polymorphism and G-banding patterns in the wild boar (Sus scrofa L) from the Netherlands. Genetica 46: 391-399. doi: 10.1007/BF00128086

3. Botstein D, White RL, Skolnick M, Davis RW. 1980. Construction of a genetic linkage map in man using restriction fragment length polymorphisms. Am J Hum Genet 32: 314-331.

4. Burgos W, Souza CA, Megens HJ, Ramayo Y, Melo M, Lemús C, Caal E, et al. 2013. Porcine colonization of the Americas: a 60k SNP story. Heredity 110:321-330. doi: 10.1038/hdy.2012.109

5. Chang WH, Chu HP, Jiang YN, Li SH, Wang Y, Chen CH, et al. 2009. Genetic variation and phylogenetics of Lanyu and exotic pig breeds in Taiwan analyzed by nineteen microsatellite markers. J Anim Sci 87: 1-8. doi:10.2527/ jas.2007-0562

6. Chapuis MP, Estoup A. 2007. Microsatellite null alleles and estimation of population differentiation. Mol Biol Evol 24: 621-631. doi:10.1093/molbev/ ms1191

7. Cortés O, Martínez AM, Cañon J, Sevane N, Gama LT, Ginja C, et al., 2016. Conservation priorities of Iberoamerican pig breeds and their ancestors based on microsatellite information. Heredity 117: 14-24. doi: 10.1038/hdy.2016.21

8. [FAO] Food and Agriculture Organization of the United Nations. 2011. Draft guidelines on molecular genetic characterization of animal genetic resources. [Internet]. Available in: http://www.fao.org/docrep/meeting/ 022/am652e.pdf

9. Giuffra E, Kijas JM, Amarger V, Carlborg O, Jeon JT, Andersson L. 2000. The origin of the domestic pig: independent domestication and subsequent introgression. Genetics 154: 1785-1791.

10. Hancock JM. 1999. Microsatellites, evolution and applications. USA: Oxford University Press. 368 p.

11. Kalinowski ST, Taper ML, Marshall TC. 2007. Revising how the computer program CERVUS accommodates genotyping error increases success in paternity assignment. Mol Ecol 16: 10991106. doi:10.1111/j.1365-294X. 2007. 03089.x

12. Kijas JM, Andersson L. 2001. A phylogenetic study of the origin of the domestic pig estimated from the nearcomplete mtDNA genome. J Mol Evol 52: 302-308. doi: 10.1007/s002390010158

13. Larson G, Dobney K, Albarella U, Fang M, Matisoo-Smith E, Robins J, Lowden S, et al. 2005. Worldwide phylogeography of wild boar reveals multiple centers of pig domestication. Science 307: 1618-1621. doi: 10.1126/ science. 1106927

14. Ministerio de Agricultura y DesarroIlo Rural. ICA. Censo pecuario nacional. Colombia. 2016. [Internet]. Disponible en: http://www.ica.gov.co/ getdoc/8232c0e5-be97-42bd-b07b$9 \mathrm{cdbfb} 07 \mathrm{fcac} /$ Censos-2012.aspx

15. Oh JD, Cacho RG, Choi JY, Seo JH, Song KD, Vega RSA, et al. 2014. Genetic analysis of Philippine native pigs (Sus scrofa L) using microsatellite loci. Philippine J Sci 143: 87-93. 
16. Peña M, Mora C. 1977. Historia de Colombia. Bogotá: Ed Norma. 270 p.

17. Pham LD, Do DN, Nam LQ, Van Ba N, Minh LT, Hoan TX, et al. 2014. Molecular genetic diversity and genetic structure of Vietnamese indigenous pig populations. J Anim Breed Genet 131: 379-386. doi: 10.1111/jbg. 12068

18. Quiroz J, Martínez, A, Marques J, Calderón J, Vega-Pla J. 2007. Relación genética de la vaca Marismeña con algunas razas andaluzas. Arch Zootec 56: 449-454.

19. Rousset F. 2008. Genepop'007: a complete re-implementation of the Genepop software for Windows and Linux. Mol Ecol Resour 8: 103-106. doi: 10.1111/ j.1471-8286.2007.01931.x

20. Sambrook J, Russell DW. 2001. Molecular cloning: a laboratory manual. $3^{\text {rd }}$ ed. New York: Cold Spring Harbor Laboratory Press. 2344 p.

21. Shanlian Q, Jichen C, Si L, Xinjian L. 2012. A comparison of silver staining protocols for detecting DNA in polyester- backed polyacrylamide gel. Braz J Microbiol 43: 649-652. doi: 10.1590/ S1517-83822012000200029

22. Szmato ${ }^{3}$ T, Ropka K, Tyra M, Piórkowska K, Zukowski K, Oczkowicz M, Blicharski T. 2016. The genetic structure of five pig breeds maintained in Poland. Ann Anim Sci 16: 1019-1027. doi: 10.1515/aoas-2016-0006

23. Wang J. 2012. On the measurements of genetic differentiation among populations. Genet Res 94:275-289. doi: 10.1017/S0016672312000481

24. Weir BS. 2012. Estimating F-Statistics: a historical view. Philos Sc. 79: 637-643. doi: 10.1086/667904

25. Zaman G, Chandra M, Laskar S, Ferdoci AM, Rank DN. 2015. Molecular characterization of Assam Local pig. Indian J Biotech 14: 416-419.

26. Zhang GX, Wang ZG, Sun FZ, Chen WS, Yang GY, Guo SJ, et al., 2003. Genetic diversity of microsatellite loci in fifty-six Chinese native pig breeds. Acta Genet Sinica 30: 225-233. 